\title{
Insomnia in Patients Suffering from Chronic Medical Illnesses: Prevalence and Impact of IAYT
}

\author{
Bista Suman1, Bhargav Praerna1, Metri Kashinath', Bhargav Hemant ${ }^{2 *}$ \\ ${ }^{1}$ S-VYASA University, Bangalore, India \\ ${ }^{2}$ NIMHANS Integrated Centre for Yoga, National Institute of Mental Health and Neurosciences, Bangalore, India \\ Email: `hemant.bhargav1@gmail.com
}

How to cite this paper: Suman, B., Praerna, B., Kashinath, M. and Hemant, B. (2017) Insomnia in Patients Suffering from Chronic Medical Illnesses: Prevalence and Impact of IAYT. Open Journal of Endocrine and Metabolic Diseases, 7, 191-201. https://doi.org/10.4236/ojemd.2017.710017

Received: September 20, 2017

Accepted: October 27, 2017

Published: October 30, 2017

Copyright $\odot 2017$ by authors and Scientific Research Publishing Inc. This work is licensed under the Creative Commons Attribution International License (CC BY 4.0).

http://creativecommons.org/licenses/by/4.0/

\begin{abstract}
Background: Sleep is an important lifestyle factor to be addressed in patients having chronic non-communicable diseases. Data revealing prevalence of insomnia in chronic medical illnesses (CMIs) in Indian population are lacking. Yoga has been found effective in improving sleep quality in patients with chronic medical illnesses (CMIs). Aim: To find the prevalence of insomnia in major chronic medical illnesses and to assess the effect of Integrated Approach of Yoga Therapy (IAYT) on them. Methods and Material: From the outpatients and inpatients of our integrative therapy clinics, 200 patients (116 males; 84 females) in the age range $49.57 \pm 11.71$ years, who satisfied the inclusion criteria and were diagnosed by the physician with any of the four major categories of CMIs: cardio-pulmonary, diabetes, musculoskeletal, and psychiatric were screened for insomnia using Insomnia Severity Index (ISI) scale. Those found suffering from clinically significant insomnia (ISI score > 14) were admitted and a residential IAYT intervention was given for a week. Before and after the intervention, ISI and Pittsburg insomnia rating scale (PIRS) were administered to assess extent of insomnia. Data was analyzed before and after the intervention using paired t-test. Results: Taking all patients of CMIs together, we found that $35 \%$ suffered from clinical insomnia, out of which, $12.5 \%$ had severe and $22.5 \%$ had moderate insomnia. We also found that prevalence of clinical insomnia was highest among those suffering from psychiatric illnesses $(62.07 \%)$ and minimum in those having musculoskeletal disorders (28.05\%). Those suffering from diabetes mellitus and cardio-pulmonary disorders reported prevalence of $32.25 \%$ and $31.94 \%$ respectively. After IAYT intervention of one week, extent of clinical insomnia reduced from $35 \%$ at the baseline to $8.5 \%$ in all patients of CMIs taken together. Following changes were observed in percentage of patients suffering from clinical insomnia in different CMIs before and after one week of IAYT interven-
\end{abstract}


tion: 1) In psychiatric patients, the percentage reduced from $62.07 \%$ to $24.13 \%$; 2) In diabetic patients, the percentage reduced from $32.27 \%$ to $3.0 \%$; 3 ) In patients having musculoskeletal disorders, the percentage reduced from $28.05 \%$ to $8.53 \%$; and 4) In patients having cardio-pulmonary illnesses, the percentage reduced from $31.94 \%$ to $2.7 \%$. Conclusion: Prevalence of insomnia is higher in patients suffering from chronic medical illnesses. IAYT intervention of one week may be helpful in reducing extent of insomnia in this population.

\section{Keywords}

Sleep, Insomnia, Chronic Medical Illnesses, Integrated Yoga

\section{Introduction}

Insomnia, or sleeplessness, is a sleep disorder in which there is an inability to fall asleep or to stay asleep as long as desired [1]. Insomnia involves difficulty in initiation, maintenance, or reduced quality of sleep. People may experience poor concentration and lower productivity as a result of insomnia [2]. Globally, prevalence of insomnia ranges from $10 \%$ to $15 \%$ among the general population with increased rates seen among older ages, female gender, white population and presence of medical or psychiatric illness [3] [4]. Panda et al. [5] found prevalence of insomnia to be $18.6 \%$ in healthy adults in South India. Another study [2], found a higher prevalence (28\%) of the same in healthy population in North India. Studies have estimated that up to $40 \%-50 \%$ of adults over the age of 60 report disturbed sleep [6].

Insomnia has also been linked to ill health. It is known that presence of chronic medical illnesses (CMIs) aggravates insomnia and those suffering from insomnia are more prone for several medical and psychiatric disorders [7]. Most chronic illnesses in modern times are life style related and sound sleep is one of the most important aspects of a healthy lifestyle. Therefore, deeper understanding and management of the problem of insomnia is of primary importance in managing chronic non-communicable diseases. A survey study on western population found that $16 \%$ of patients with CMIs had severe and $34 \%$ had mild insomnia at baseline [7]. Such data in chronically ill Indian population are lacking.

Medications for insomnia appear to cause both psychological dependence and physical dependence; they may also cause cognitive decline along with morning sedation [8]. Sleep is affected by variety of factors, starting from the change in surroundings, to diet, to the deep psychological conflicts, therefore, it is very important to use a holistic comprehensive lifestyle intervention such as Yoga based lifestyle to address this issue. Yoga is one of the spiritual practices derived from the orthodox school of Hindu philosophy. Yoga is a science of Holistic living and not merely a set of physical postures (asanas) and breathing techniques (pranayama). It is a psycho-physical and spiritual science, which aims at the harmonious development of the human body, mind and soul [9]. The Integrated 
Approach of Yoga Therapy (IAYT) is an approach which consists in not only dealing with physical layer but also includes using different yoga techniques to operate on different layers of our existence: the physical layer, the layer of prana, the mental layer, the layer of wisdom, and the layer of bliss [10].

Several studies have been conducted on the effect of yoga in insomnia. Almost all the studies have showed positive effects of yoga on insomnia [11]. As yoga has been shown to be effective in management of both insomnia as well as chronic diseases, IAYT might be an effective treatment option for insomnia in patients suffering from chronic medical illnesses [12]. Present study was planned with an intention to know the prevalence of insomnia in patients of different chronic diseases in India and the effect of IAYT on them.

\section{Methods}

Two hundred patients (116 males; 84 females) in the age range $49.57 \pm 11.71$ years, who were diagnosed by physician to be suffering from any of the four major categories of CMIs: cardio-pulmonary; diabetes; musculoskeletal; and psychiatric were included in the study. They were screened from the outpatients and inpatients departments of our integrative therapy clinics. The inclusion requirements were: a) those that may or may not be using medications for sleep; $b$ ) those with mild and moderate severity of illness; c) ambulatory patients and d) those having knowledge of English language. The exclusion criteria were as follows: a) those with severe and complicated CMIs and bed ridden patients a) Acute injury within the past 6 months; b) Inability to stand without assistance; c) More than 6 sessions of yoga practice within the past 3 months; d) Those in whom sleep medication was changed within the past 1 month; e) Untreated or poorly managed medical diagnosis; f) Those with complications and severe medical illnesses; g) Diagnosis of clinical signs of a primary sleep disorder (e.g., sleep apnea, restless legs syndrome; h) Consumption of caffeine equivalent of $>3$ cups coffee/day or alcohol $>2$ drinks/day at least 4 days/week.

Written informed consent was taken from all the subjects before the study and Institutional Ethical Clearance was obtained.

Single group pre-post design was followed to assess the effect of one week IAYT intervention in those suffering from clinical insomnia. Screening was done using a validated and reliable screening tool, the Insomnia Severity Index (ISI) scale. Those found suffering from clinically significant insomnia (ISI score > 14) were admitted and a residential IAYT intervention was given for a week. Before and after the intervention, ISI and Pittsburg insomnia rating scale (PIRS) were administered to assess extent of insomnia. Data was collected on the first day of admission to the one week residential yoga therapy program and was collected again at the end of one week. Subjects were given orientation to yoga therapy program and the research work on the first day and after this data collection was started. Both pre and post data were collected on the same time of the day. Subjects were explained the details of the questionnaire and instructions were given clearly before filling up the questionnaires. 
All data were double-entered in Microsoft Excel and analyzed using Statistical Package for the Social Sciences (SPSS) version 16.0. Pre- and post-intervention scores on the questionnaires had been compared using paired t-tests (and Wilcoxon signed-ranks tests for skewed data). All tests were two-tailed. Given the exploratory nature of the study, a significance level of 0.05 was set for all tests.

\section{Assessments}

\subsection{Insomnia Severity Index (ISI)}

The ISI is a valid and reliable screening tool to assess and diagnose the severity of insomnia of the patients [13]. It consists of 7 questions concerning sleep onset, sleep maintenance, early awakening, level of satisfaction with sleep pattern, extent of interference with daily functioning, conspicuousness of impairment caused by sleep problem, and level of concern about current sleep problem. Each item is marked on a 5-point Likert scale (0 to 4 ). Total scores after evaluation range from 0 to 28; higher the score more severe is the insomnia. Scores 0 to 7 indicate no clinically significant insomnia, 8 to 14 sub-threshold insomnia, 15 to 21 clinically significant insomnia (moderate), and 22 to 28 clinically significant insomnia (severe).

\subsection{Pittsburg Insomnia Rating Scale}

Cronbach's alpha coefficient for Total PIRS score is 0.93 which indicates excellent internal consistency [14]. It is a scale with 65-items. Subjects score the items that have three broad sections. Initial is the subjective distress score (46 items), then subjective sleep parameters (10 items) and last is the quality-of-life (9 items). Section A of the scale has a $10 \mathrm{~cm}$ line to mark the quality of sleep in the past week. This answer is not used in the scoring. Section B has 46 questions which have to be answered on the Likert scale from 0 - $3(0=$ not at all bothered, 1 = slightly bothered, 2 = moderately bothered, 3 severely bothered). It is scored by adding all the answers. This is the distress score. Section $\mathrm{C}$ has 10 questions which have to be answered on the Likert scale 0 - 3 with variable answers depending on the question. Score of this section is the addition of all and is termed as sleep parameters score. Section D has 9 questions which have to be answered on the Likert scale from 0 - $3(0=$ excellent, $1=$ good, $2=$ fair, $3=$ poor $)$. Addition of all the answers gives the final score which is termed as Quality of life score. Section $\mathrm{E}$ is about comments which the patient wants to put in but it is not included in the scoring. Final score is the grand total of all the three components. Minimum score is 0 (good) and maximum is 195 (bad) [15].

\section{Intervention}

\section{Integrated Approach of Yoga Therapy (IAYT)}

IAYT is based on the basic principle that there are 5 layers of the existence to human beings namely Annamaya Kosa (physical level), Pranamaya Kosa (subtle 
energy level), Manomaya Kosa (emotional level), Vijnanamaya Kosa (level of intellect) and Anandamaya Kosa (level of bliss). Yogic pathophysiology propounds that the disturbances at the emotional level ( $a d h i)$ percolate to the physical level ( vyadhi) through the layer of prana. Furthermore, all layers are interrelated and they affect each other indirectly. The IAYT is an approach which consists in not only dealing with physical layer but also includes using techniques to operate on different layers of our existence. The practices at annamaya kosa include yogasanas, loosening practices, yoga diet and yoga kriyas, at pranamaya kosa-breathing practices, breathing kriyas and pranayama. At manomaya kosa, the practices are meditations (cyclic meditation, om meditation), devotional sessions and happy assembly, where as lectures, counseling and satsangs correct the notions about one's life ambitions and goals at vijnanmaya kosha that form the basic conceptual root for the life style of the individual. Karma yoga and tuning to nature are the practices that help one to get established in anandamaya kosa bringing bliss in our lives [10]. Based on the above mentioned concepts holistic IAYT module was made and patients followed the yoga based lifestyle routine given in Table 1.

\section{Results}

Data for all the variables was found normally distributed by Shapiro-wilk test

Table 1. Yoga based lifestyle routine under IAYT intervention.

\begin{tabular}{|c|c|}
\hline Time & Schedule \\
\hline 05:30 am & Pranayama, Om meditation \\
\hline 06:30 am & Yogasanas session 1 (tailor made based on individual complaints) \\
\hline 07:30 am & Breakfast \\
\hline 08:00 am & Lecture on lifestyle advises as per Bhagawad Gita \\
\hline 09:00 am & Consultation with Integrative Medicine Physicians \\
\hline 09:45 am & Yogic Counseling \\
\hline $12: 15$ am & Lecture on Stress Management: yogic and modern concepts \\
\hline 01:00 am & Lunch \\
\hline 02:00 am & Karma Yoga \\
\hline 03:00 am & Cyclic Meditation \\
\hline 04:00 am & Yogasanas session 1 (tailor made based on individual complaints) \\
\hline 05:00 am & Walk and tuning to nature \\
\hline 06:00 am & Bhajan (devotional sessions) \\
\hline $06: 30$ am & Trataka, MSRT (advanced meditations) \\
\hline 07:30 am & Dinner \\
\hline 08:15 am & Group discussions and introspection for the day \\
\hline $10: 00 \mathrm{am}$ & Lights off \\
\hline
\end{tabular}


except for the variable Insomnia Severity Scale Scores (ISI Scores).

\subsection{Overall Prevalence}

All chronic medical illnesses taken together we found that out of 200 subjects, 70 (35\%) were suffering from clinical insomnia (ISI score $>14$ ), out of which, 25 (12.5\%) had severe insomnia (ISI score between 22 - 28) and 45 (22.5\%) had moderate insomnia (ISI score between 15 - 21). Another 49 (29.5\%) patients had subclinical insomnia (ISI score between 8 - 14).

\subsubsection{Prevalence of Insomnia in Diabetic Patients}

Out of all 200 subjects, 31 suffered from diabetes. Out of 31 diabetic subjects, 10 (32.25\%) had clinical insomnia (ISI score > 14), out of which $2(6.45 \%)$ had severe insomnia (ISI score between 22 - 28) and 8 (25.8\%) were suffering from moderate insomnia (ISI score between 15 - 21).

\subsubsection{Prevalence of Insomnia in Patients Suffering Musculoskeletal Disorders}

Out of total 200 subjects, 82 suffered from musculoskeletal diseases. We found that out of 82 subjects, 23 (28.05\%) had clinical insomnia (ISI score $>14$ ), out of which 9 (10.98\%) suffered from severe insomnia (ISI score between 22 - 28) and $14(17.07 \%)$ suffered from moderate insomnia (ISI score between 15 - 21).

\subsubsection{Prevalence of Insomnia in Psychiatric Illnesses}

Twenty nine out of 200 subjects suffered from psychiatric illnesses. Out of 29 subjects, 18 (62.07\%) had clinical insomnia (ISI score > 14), among them, 7 (24.14\%) were suffering from severe insomnia (ISI score between 22 - 28) and 11 (37.93\%) were having moderate insomnia (ISI score between 15 - 21).

\subsubsection{Prevalence of Insomnia in Patients Suffering from Cardio-Pulmonary Diseases}

Cardiopulmonary disorders were diagnosed in 72 out of 200 subjects of CMIs. Among the total 72 subjects, 23 (31.94\%) had clinical insomnia (ISI score > 14), where 7 (9.72\%) had severe insomnia (ISI score between 22 - 28) and 16 (22.22\%) had moderate insomnia (ISI score between 15 - 21).

\subsection{Within Group Comparisons}

\subsubsection{Overall Comparison}

It was found that overall prevalence of clinical insomnia reduced from 35\% at the baseline to $8.5 \%$ at the end of one week of IAYT intervention. Following changes were observed in prevalence of clinical insomnia in different CMIs before and after one week of IAYT intervention: 1) In psychiatric patients, the prevalence reduced from $62.07 \%$ to $24.13 \%$; 2) In diabetic patients the prevalence reduced from $32.2 .7 \%$ to $3.0 \%$; 3 ) In patients having musculoskeletal disorders the prevalence reduced from $28.05 \%$ to $8.53 \%$; and 4) In patients having cardio-pulmonary illnesses the prevalence reduced from $31.94 \%$ to $2.7 \%$. 


\subsubsection{Overall Comparison of Sleep Variables in Subjects Suffering from Chronic Medical Illnesses with Moderate Clinical Insomnia}

In subjects having moderate clinical insomnia (ISI Score between 15 - 21), within group comparison of the variables before and after one week of IAYT intervention (using paired $t$ test or wilcoxon signed rank test) revealed that there was a highly significant improvement in Insomnia Severity Index $(\mathrm{p}=0.001)$ and Pittsburg Insomnia Rating Scale (PIRS) Total Score ( $\mathrm{p}=0.001$; see Table 2). Independent analysis of different subsets of PIRS also showed highly significant improvement in Subjective Distress Scores (PIRS_SDS; $\mathrm{p}=0.001$ ), Subjective Sleep Parameter (PIRS_SSP; $\mathrm{p}=0.001$ ) and Quality of Life (PIRS_QOL; $\mathrm{p}$ $=0.001$ ).

\subsubsection{Overall Comparison of Sleep Variables in Subjects Suffering from Chronic Medical Illnesses with Severe Clinical Insomnia}

In subjects having severe clinical insomnia (ISI Score between 22 - 28), within group comparison of the variables before and after one week of IAYT intervention (using paired t test or wilcoxon signed rank test) revealed that there was a highly significant improvement in Insomnia Severity Index $(\mathrm{p}=0.001)$ and Pittsburg Insomnia Rating Scale (PIRS) Total Score ( $p=0.001$; see Table 3). Independent analysis of different subsets of PIRS also showed highly significant improvement in Subjective Distress Scores (PIRS_SDS; $\mathrm{p}=0.001$ ), Subjective Sleep Parameter (PIRS_SSP; $\mathrm{p}=0.001$ ) and Quality of Life (PIRS_QOL; $\mathrm{p}=$ $0.001)$.

\section{Discussion}

It is known that presence of chronic medical illnesses aggravates insomnia and those suffering from insomnia are more prone for several medical and psychiatric disorders. A survey study on patients with chronic illness found, on the basis of their working definition of insomnia, $16 \%$ of study patients had severe and 34\% had mild insomnia at baseline (Katz, \& McHorney, 1998). Another western

Table 2. Table showing paired comparison of sleep variables in subjects suffering from chronic medical illnesses with moderate clinical insomnia.

\begin{tabular}{|c|c|c|c|c|c|c|c|c|}
\hline \multirow[t]{2}{*}{ SN } & \multirow[t]{2}{*}{ Variable } & \multirow[t]{2}{*}{$\mathrm{n}$} & \multirow{2}{*}{$\begin{array}{c}\text { Mean } \pm \text { SD } \\
\text { Pre }\end{array}$} & \multirow{2}{*}{$\begin{array}{c}\text { Mean } \pm \text { SD } \\
\text { Post }\end{array}$} & \multicolumn{2}{|c|}{$\begin{array}{c}\text { 95\% confidence } \\
\text { interval }\end{array}$} & \multirow{2}{*}{$P$ value } & \multirow{2}{*}{$\begin{array}{c}\% \\
\text { improvement }\end{array}$} \\
\hline & & & & & Upper & Lower & & \\
\hline 1 & ISI Score & 45 & $16.33 \pm 1.51$ & $9.02 \pm 3.82$ & 8.32 & 6.30 & $0.00^{\star \star a}$ & 44.76 \\
\hline 2 & PIRS_SD & 45 & $69.62 \pm 22.32$ & $38.53 \pm 17.97$ & 36.55 & 25.63 & $0.00^{\star * b}$ & 44.66 \\
\hline 3 & PIRS_SSP & 45 & $13.18 \pm 5.19$ & $7.07 \pm 4.46$ & 7.57 & 4.65 & $0.00^{\star * b}$ & 46.36 \\
\hline 4 & PIRS_QOL & 45 & $16.64 \pm 5.00$ & $10.49 \pm 5.09$ & 7.66 & 4.65 & $0.00^{\star * b}$ & 36.96 \\
\hline 5 & PIRS_TOTAL & 45 & $99.44 \pm 28.74$ & $56.09 \pm 23.70$ & 50.76 & 35.95 & $0.00^{\star \star b}$ & 43.59 \\
\hline
\end{tabular}

${ }^{* *} \mathrm{p}<0.01$. ${ }^{\mathrm{a} W}$ ilcoxon related samples signed rank test. ${ }^{\mathrm{b}}$ Paired samples $\mathrm{t}$ test. Abbreviations: ISI: Insomnia severity index; PIRS_SD: Pittsburg insomnia rating scale_Subjective distress scores; PIRS_SS: Pittsburg insomnia rating scale_Subjective sleep scores; PIRS_QOL: Pittsburg insomnia rating scale_Quality of Life; PIRS_TOTAL: Pittsburg insomnia rating scale_Total scores. 
Table 3. Table showing paired comparison of sleep variables in subjects suffering from chronic medical illnesses with severe clinical insomnia.

\begin{tabular}{|c|c|c|c|c|c|c|c|c|}
\hline \multirow{3}{*}{ SN } & \multirow{3}{*}{ Variable } & \multirow{3}{*}{$\mathrm{n}$} & \multirow{3}{*}{$\begin{array}{c}\text { Mean } \pm \text { SD } \\
\text { Pre }\end{array}$} & \multirow{3}{*}{$\begin{array}{c}\text { Mean } \pm \text { SD } \\
\text { Post }\end{array}$} & \multirow{2}{*}{\multicolumn{2}{|c|}{$\begin{array}{c}95 \% \text { confidence } \\
\text { interval } \\
\end{array}$}} & \multirow{3}{*}{$P$ value } & \multirow{3}{*}{$\begin{array}{c}\% \\
\text { improvemen }\end{array}$} \\
\hline & & & & & & & & \\
\hline & & & & & Upper & Lower & & \\
\hline 1 & ISI Score & 25 & $24.12 \pm 2.37$ & $13.56 \pm 5.52$ & 12.74 & 8.38 & $0.00^{* \star a}$ & 43.78 \\
\hline 2 & PIRS_SD & 25 & $89.40 \pm 25.41$ & $58.68 \pm 30.44$ & 40.67 & 20.77 & $0.00^{\star * b}$ & 34.36 \\
\hline 3 & PIRS_SSP & 25 & $20.04 \pm 6.13$ & $12.44 \pm 6.56$ & 10.24 & 4.96 & $0.00^{\star * b}$ & 37.92 \\
\hline 4 & PIRS_QOL & 25 & $19.76 \pm 5.24$ & $13.60 \pm 6.36$ & 8.49 & 3.83 & $0.00^{* * b}$ & 31.17 \\
\hline 5 & PIRS_TOTAL & 25 & $129.20 \pm 30.89$ & $84.72 \pm 40.42$ & 57.40 & 31.56 & $0.00^{* * \mathrm{~b}}$ & 34.43 \\
\hline
\end{tabular}

${ }^{* *} \mathrm{p}<0.01$. ${ }^{\mathrm{a} W i l c o x o n}$ related samples signed rank test. ${ }^{b}$ Paired samples $\mathrm{t}$ test. Abbreviations: ISI: Insomnia severity index; PIRS_SD: Pittsburg insomnia rating scale_Subjective distress scores; PIRS_SS: Pittsburg insomnia rating scale_Subjective sleep scores; PIRS_QOL: Pittsburg insomnia rating scale_Quality of Life; PIRS_TOTAL: Pittsburg insomnia rating scale_Total scores.

study had shown that people with chronic insomnia reported more of the following than did people without insomnia: heart disease (21.9\% vs. $9.5 \%)$, high blood pressure ( $43.1 \%$ vs. $18.7 \%)$, neurologic disease (7.3\% vs. $1.2 \%)$, breathing problems $(24.8 \%$ vs. $5.7 \%)$, urinary problems ( $19.7 \%$ vs. $9.5 \%)$, chronic pain (50.4\% vs. $18.2 \%)$, and gastrointestinal problems (33.6\% vs. $9.2 \%)$ and conversely, people with the following medical problems reported more chronic insomnia than did those without those medical problems: heart disease (44.1\% vs. $22.8 \%)$, cancer $(41.4 \%$ vs. $24.6 \%)$, high blood pressure $(44.0 \%$ vs. $19.3 \%)$, neurologic disease $(66.7 \%$ vs. $24.3 \%)$, breathing problems (59.6\% vs. $21.4 \%)$, urinary problems $(41.5 \%$ vs. $23.3 \%)$, chronic pain $(48.6 \%$ vs. $17.2 \%)$, and gastrointestinal problems (55.4\% vs. $20.0 \%)$ [16]. Insomnia is also a core symptom in a range of chronic pain conditions with less certain organic etiology, such as fibromyalgia and other musculoskeletal disorders [17].

As compared to the above results we found overall prevalence of clinical insomnia in four major types of chronic medical illnesses (cardio-pulmonary diseases, diabetes, chronic musculoskeletal illnesses and psychiatric illnesses) to be $35 \%$ out of which $12.5 \%$ had severe insomnia and $22.5 \%$ had moderate insomnia. These results are similar to those obtained in a previous study where $16 \%$ had chronic insomnia and 34\% had mild insomnia [7].

We also found that prevalence of clinical insomnia varied across different types of chronic medical illnesses with highest prevalence among those suffering from psychiatric illnesses (62.07\%) and minimum prevalence in those having musculoskeletal disorders (28.05\%). Those suffering from diabetes mellitus type 2 had second highest prevalence (32.25\%) and those having cardio-pulmonary disorders reported prevalence of clinical insomnia to be $31.94 \%$.

We found that prevalence of severe insomnia was also highest among patients with psychiatric illnesses (24.14\%). This was followed by those with chronic musculoskeletal disorders (10.98\%), cardiopulmonary disorders (9.72\%) and then those with diabetes (6.45\%). Previously, similar western study had shown 
that people with the following medical problems reported more chronic insomnia: heart disease (44.1\%), cancer (41.4\%), high blood pressure (44.0\%), neurologic disease (66.7\%), breathing problems (59.6\%), urinary problems $(41.5 \%)$, chronic pain (48.6\%), and gastrointestinal problems (55.4\%) (Taylor et al., 2007). Taylor et al. found the prevalence to be highest in those suffering from neurological disorders. Unfortunately, we did not measure the same in this population due to lack of sufficient number of patients. We found prevalence of clinical insomnia to be $31.94 \%$ in patients in cardio-pulmonary diseases, which is lower than that reported by Taylor et al. (44.1\% to 59.6\%). [17] We find that our overall prevalence rates of insomnia are on a lower side as compared to those found by Taylor et al., this may be because they included patients suffering from severe chronic illness, which we excluded. Taylor et al. did not include those suffering from psychiatric illnesses in their study, which we included and found the prevalence to be much higher.

No previous study has seen the impact of a week of IAYT intervention on insomnia in various chronic medical illnesses before. Ours is the first study to report this and we have shown highly significant impact of a holistic yoga intervention on insomnia in as lesser a duration as 1 week, we found that as per ISI score clinical insomnia $(>14)$ prevalence which was $35 \%$ at the baseline came down to $8.5 \%$ after one week of IAYT intervention.

We found that overall prevalence of insomnia is much higher in those having chronic medical illnesses as compared to healthy adults (35\% vs. $18 \%$ ). Among those with chronic medical illnesses (CMIs) we found insomnia rate and severity was highest among those with psychiatric disorders. Though there are not many studies which looked into prevalence of insomnia in psychiatric illnesses before, a couple of studies have done so, but they are conducted in western countries and report a much lower prevalence than what we found [18] [19]. This may be because of change in environmental conditions and socio-cultural values. Higher prevalence of insomnia in those having CMI may be because of various causes depending on type of CMI. For e.g., in psychiatric patients it may be because of inherent nature of psychiatric disorder which involves disturbances of circadian rhythm due to imbalanced neurotransmitter release; in patients with diabetes it may be due to increase frequency of micturition during night time; in patients with cardio-pulmonary disorders it may be due to breathlessness; in patients with musculoskeletal disorders it may be due to pain and deformities.

IAYT intervention reduced the overall prevalence of insomnia from $35 \%$ to $8.5 \%$. This is a very significant change. The probable reason may be adjustment of the lifestyle, removal of stressor from the environment, increased cardiopulmonary efficiency and control of sugar levels in diabetics due to increased physical activity and reduction of stress. Overall, IAYT brings deep relaxation to the system through parasympathetic dominance which is a promoter of sleep. Through yogic counseling deep rooted stressors in sub conscious mind are identified and deeper levels of relaxation are reached. This may drastically reduced 
the prevalence of insomnia.

This is a preliminary study to investigate the relation between CMIs and insomnia and pilot attempt to understand the role of IAYT intervention in addressing insomnia in CMIs. Lack of control group limits generalizability of the results. In future, larger randomized controlled trials should be conducted in each CMI exclusively with large sample size to test the efficacy of IAYT. Future studies should also do longer follow ups to see whether the effects are maintained for a longer time with IAYT practices.

\section{Conclusion}

Prevalence of insomnia is higher in patients suffering from chronic medical illnesses. IAYT intervention of one week may be helpful in reducing prevalence of insomnia in this population. Future studies should focus on testing the efficacy of IAYT in each category of CMI separately on using a larger sample size and randomized controlled design.

\section{Acknowledgements}

We acknowledge the subjects who gave their consent and participated in this study.

\section{References}

[1] Golub, R.M. (2012) Insomnia. JAMA: The Journal of the American Medical Association, 307, 2653-2653.

[2] Nilesh, S., Abha, B. and Arpina, B. (2010) Indian Research on Sleep Disorders. Indian Journal of Psychiatry, 52, S255-S259. https://doi.org/10.4103/0019-5545.69242

[3] Saddichha, S. (2010) Disease Mongering in Psychiatry: Fact or Fiction? JNMA: The Journal of the American Medical Association, 50, 320-327. https://doi.org/10.2202/1948-4682.1042

[4] de Souza Lopes, C., Robaina, J.R. and Rotenberg, L. (2012) Epidemiology of Insomnia: Prevalence and Risk Factors. In Can't Sleep? Issues of Being an Insomniac. InTech, 1, 3-22.

[5] Panda, S., Taly, A.B., Sinha, S., Gururaj, G., Girish, N. and Nagaraja, D. (2012) Sleep-Related Disorders among a Healthy Population in South India. Neurology India, 60, 68. https://doi.org/10.4103/0028-3886.93601

[6] Roepke, S.K. and Ancoli, I. (2010) Sleep Disorders in the Elderly. Indian Journal of Medical Research, 131, 301-302.

[7] Katz, D.A. and McHorney, C.A. (1998) Clinical Correlates of Insomnia in Patients with Chronic Illness. Archives of Internal Medicine, 158, 107-1099.

[8] Carlstedt, R.A. (2012) Evidence-Based Applied Sport Psychology: A Practitioner's Manual. Springer Publishing Company.

[9] Bhobe, S. (2012) Integrated Approach to Yoga. The Nursing Journal of India, 91, 33-42.

[10] Nagendra, H.R. and Nagarathna, R. (2004) Promotion of Positive Health. Swami Vivekananda Yoga Prakashana, Bangalore, India.

[11] Afonso, R.F. (2012) Yoga Decreases Insomnia in Postmenopausal Women: A Ran- 
domized Clinical Trial. Menopause, 19, 186-193. https://doi.org/10.1097/gme.0b013e318228225f

[12] Sobana, R., Parthasarathy, S., Du Rai Samy, K.J. and Vadivel, S. (2013) The Effect of Yoga Therapy on Selected Psychological Variables among Male Patients with Insomnia. Journal of Clinical and Diagnostic Research: JCDR, 7, 55. https://doi.org/10.7860/JCDR/2012/5056.2669

[13] Bastien, C.H., Annie, V. and Charles, M.M. (2001) Validation of the Insomnia Severity Index as an Outcome Measure for Insomnia Research. Sleep Medicine, 2, 297-307. https://doi.org/10.1016/S1389-9457(00)00065-4

[14] Veqar, Z., Jamal, A.M. and Mahammed, E.H. (2014) Psychometric Analysis of the Pittsburgh Insomnia Rating Scale among University Population of Poor Sleepers in India. North American Journal of Medical Sciences, 6, 161. https://doi.org/10.4103/1947-2714.131238

[15] Morin, C.M., Belleville, G., Bélanger, L. and Ivers, H. (2011) The Insomnia Severity Index: Psychometric Indicators to Detect Insomnia Cases and Evaluate Treatment Response. Sleep, 34, 601. https://doi.org/10.1093/sleep/34.5.601

[16] Taylor, D.J., Mallory, L.J., Lichstein, K.L., Durrence, H., Riedel, B.W. and Bush, A.J. (2007) Comorbidity of Chronic Insomnia with Medical Problems. Sleep, 30, 213. https://doi.org/10.1093/sleep/30.2.213

[17] Moldofsky, H. (2001) Sleep and Pain. Sleep Medicine Reviews, 5, 385-396. https://doi.org/10.1053/smrv.2001.0179

[18] Breslau, N., Roth, T., Rosenthal, L. and Andreski, P. (1996) Sleep Disturbance and Psychiatric Disorders: A Longitudinal Epidemiological Study of Young Adults. Biological Psychiatry, 39, 411-418. https://doi.org/10.1016/0006-3223(95)00188-3

[19] Ford, D.E. and Kamerow, D.B. (1989) Epidemiologic Study of Sleep Disturbances and Psychiatric Disorders: An Opportunity for Prevention? JAMA, 262, 1479-1484. https://doi.org/10.1001/jama.1989.03430110069030 